ARTICLE

Received 1 Oct 2016 | Accepted 21 Dec 2016 | Published 22 Feb $2017 \quad$ DOl: 10.1038/ncomms14380 OPEN

\title{
Plasmon-assisted high-harmonic generation in graphene
}

Joel D. Cox ${ }^{1}$, Andrea Marini ${ }^{1} \&$ F. Javier García de Abajo ${ }^{1,2}$

High-harmonic generation in condensed-matter systems is both a source of fundamental insight into quantum electron motion and a promising candidate to realize compact ultraviolet and ultrafast light sources. While graphene is anticipated to efficiently generate high-order harmonics due to its anharmonic charge-carrier dispersion, experiments performed on extended samples using $\mathrm{THz}$ illumination have revealed only a weak effect. The situation is further complicated by the enormous electromagnetic field intensities required by this highly nonperturbative nonlinear optical phenomenon. Here we argue that the large light intensity required for high-harmonic generation to occur can be reached by exploiting localized plasmons in doped graphene nanostructures. We demonstrate through rigorous time-domain simulations that the synergistic combination of strong plasmonic near-field enhancement and a pronounced intrinsic nonlinearity result in efficient broadband high-harmonic generation within a single material. Our results support the strong potential of nanostructured graphene as a robust, electrically tunable platform for high-harmonic generation.

\footnotetext{
${ }^{1}$ ICFO-Institut de Ciencies Fotoniques, The Barcelona Institute of Science and Technology, 08860 Castelldefels (Barcelona), Spain. ${ }^{2}$ ICREA-Institució Catalana de Recerca i Estudis Avançats, Passeig LLuís Companys 23, 08010 Barcelona, Spain. Correspondence and requests for materials should be addressed to J.D.C. (email: joel.cox@icfo.es) or to A.M. (email: andrea.marini@icfo.es) or to F.J.G.d.A. (email: javier.garciadeabajo@icfo.es).
} 
H igh-harmonic generation (HHG) is an extreme nonlinear optical phenomenon first observed by driving atomic gases with intense ultrashort light pulses ${ }^{1-3}$. The harmonic intensity remains surprisingly large up to a high order of the pulse carrier frequency, stimulating applications for HHG as a source of ultraviolet and X-ray radiation ${ }^{4-6}$, as well as in the generation of attosecond pulses ${ }^{7-9}$, which has enabled tomographic imaging of molecular orbitals ${ }^{10}$ and the exploration of subfemtosecond dynamics in chemical reactions ${ }^{11}$.

Recent observations of HHG from condensed-matter systems ${ }^{12-16}$ are currently attracting much interest not only in the pursuit of new solid-state optical technologies, but also in the underlying physics of HHG in bulk crystals and its analogy with atomic gases. Indeed, while HHG from individual atoms is well understood as the coherent emission produced by the optically induced tunneling ionization of an electron, its acceleration by the driving field, and the subsequent recollision with its parent ion $^{17,18}$, the picture becomes less clear in crystalline media, where collective effects associated with the high density of electrons and their interaction with the lattice significantly complicate the generation process. As expected, HHG in solids is found to depend strongly on the electronic band structure and the interplay between inter- and intraband transitions ${ }^{12,13,15,19-21}$

The linear, gapless dispersion relation of graphene electrons ${ }^{22,23}$ anticipates a strong nonlinear optical response of the atomically thin material, which recent experiments confirm to be intrinsically large ${ }^{24-29}$. On the theory side, monolayer graphene is expected to produce intense $\mathrm{HHG}$ in the $\mathrm{THz}$ regime ${ }^{30,31}$, attributed to complementary inter- and intraband charge-carrier motion at low temperatures and doping levels. Unfortunately, recent experiments report either no evidence ${ }^{32}$ or only a weak effect ${ }^{33}$ associated with the generation of low-order harmonics from multilayer graphene for currently available $\mathrm{THz}$ illumination intensities. This situation could be improved by using more intense sources at higher frequencies, and further relying on enhanced graphene-light interaction mediated by localized plasmon resonances.
When resonantly driven by optical fields, plasmons, the collective oscillations of electrons in conducting media, concentrate the incident electromagnetic energy into extremely subwavelength volumes, generating intense local electric fields that are essential to trigger nonlinear optical phenomena. In graphene, plasmons offer an efficient way to couple the atomically thin carbon layer with impinging light ${ }^{34-40}$, while their associated near-field enhancement, in combination with the highly anharmonic response of graphene $31,41,42$, is predicted to give rise to large optical nonlinearies ${ }^{43-47}$. Importantly, these plasmons only exist in highly doped graphene, while their frequency is strongly dependent on the doping level ${ }^{34-36,39,40}$. Electrical gating thus provides a mechanism to tune the harmonic generation in graphene to the desired frequency range.

Here we predict that highly efficient HHG takes place in doped graphene nanostructures when the incident light is tuned to their localized plasmons. Specifically, we obtain harmonic intensities that are orders of magnitude higher than in other materials. In addition, no sharp cutoff is observed with harmonic order. Our results are based on nonperturbative time-domain numerical simulations of the nonlinear optical response of graphene using two complementary approaches: a random-phase approximation (RPA) description of the single-particle density matrix within a tight-binding (TB) model for the electrons of ribbons and finite islands ${ }^{43}$; and the solution of the single-particle Bloch equations for massless Dirac-fermions (MDFs) in extended graphene, complemented by a classical electromagnetic (CEM) description of the self-consistent field produced by the illuminated nanostructure (see Methods). We find both approaches to be in excellent agreement at intensities below the saturable absorption threshold. Our prediction of highly efficient HHG assisted by coupling to graphene plasmons suggests applications to a wide range of nonlinear photonic technologies, including tunable sources of broadband attosecond light. a

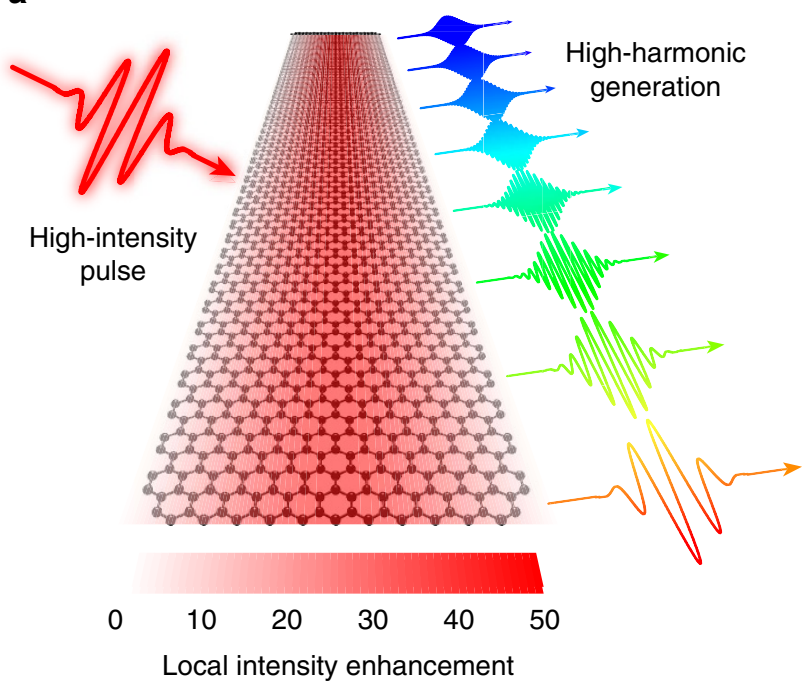

b
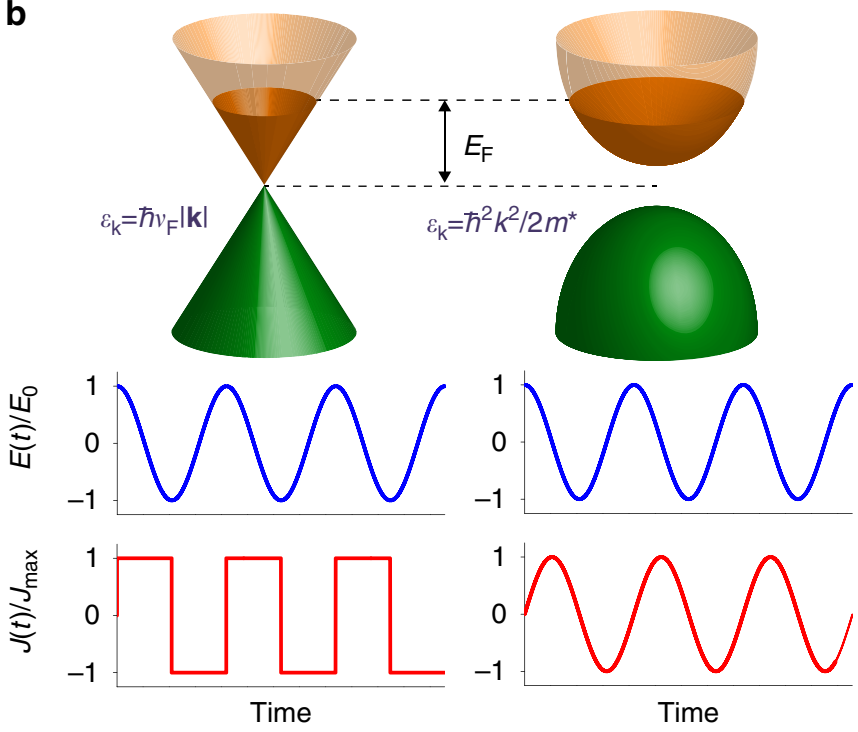

Figure 1 | High-harmonic generation (HHG) assisted by graphene plasmons. (a) Schematic illustration of a doped graphene nanoribbon illuminated by an intense optical pulse that is resonant with the ribbon transverse dipole plasmon. The latter produces strong in-plane electric-field intensity enhancement (see colour scale) that boosts the generation of high harmonics. (b) The low-energy band structures of graphene (upper left) and a conventional 2D semiconducting crystal (upper right) respond differently to a monochromatic light electric field $E(t)=E_{0} \cos (\omega t)$ : in graphene, the induced current $J(t)$ (lower left) acquires a square-wave temporal profile in the high-intensity limit, which contains all odd-order harmonics in its Fourier decomposition, while the semiconductor responds harmonically at the driving frequency $\omega$. A 2D free-electron gas also shows a harmonic response ${ }^{52}$. 


\section{Results}

Extreme nonlinear optics in doped graphene. In practice, cumbersome laser amplification schemes are usually needed to reach the extreme electromagnetic field intensities required to generate high-order harmonics. To overcome this limitation, plasmonic nanostructures have attracted considerable interest as in situ electric field enhancers for HHG in gaseous media ${ }^{48-51}$. As illustrated schematically in Fig. 1a, we propose that compact, efficient HHG can be realized in graphene by combining the intense near-field enhancement associated with graphene plasmons and the intrinsically high nonlinear optical response of this material. The appeal of graphene as a nonlinear optical material stems in part from its linear charge-carrier dispersion with electron wave vector $\mathbf{k}$ at low energies, $\varepsilon_{\mathbf{k}}=\hbar v_{\mathrm{F}}|\mathbf{k}|$, where $v_{\mathrm{F}} \approx c / 300$ is the Fermi velocity. In the single-particle MDF description of doped monolayer graphene, neglecting interband electronic transitions, this linear dispersion relation leads to a maximum achievable surface current density $J_{\max }=-e n v_{\mathrm{F}}$ $\operatorname{sign}\{\sin (\omega t)\}$ when illuminated by a monochromatic field $E(t)=E_{0} \cos (\omega t)$ in the $E_{0} \rightarrow \infty$ limit $^{30,41}$. The current is thus limited by the doping charge-carrier density $n$. This square-wave profile of the induced current density under intense illumination translates into efficient generation of odd-ordered harmonics (Fig. 1b). Conversely, in conventional two-dimensional (2D) media, for which charge carriers obey a parabolic dispersion relation $\varepsilon_{\mathbf{k}}=\hbar^{2} k^{2} / 2 m^{*}$, the system responds harmonically at the frequency of the driving field, regardless of electron-electron interactions ${ }^{52}$. While this comparison favourably portrays graphene as a highly nonlinear optical material, it is important to note that interband optical transitions become significant at high intensities, even when the system is driven at frequencies below the Fermi level ${ }^{30}$.

Plasmon-assisted HHG from graphene nanoribbons. Quantitative analysis of plasmon-enhanced HHG in a doped graphene nanoribbon is presented in Fig. 2. The linear optical absorption of the nanoribbon under consideration $\left(20 \mathrm{~nm}\right.$ width, $E_{\mathrm{F}}=0.4 \mathrm{eV}$
Fermi energy) shows a prominent dipolar plasmon (Fig. 2a), as predicted by TB-RPA atomistic simulations and classical electrodynamics, in excellent mutual agreement. We thus consider HHG produced by incident pulses with central frequency tuned to that plasmon. We present HHG simulations obtained with the MDF-CEM and TB-RPA approaches (see Methods) in Fig. 2b, which shows the spectral decomposition (time-Fourier transform) of the radiative emission intensities for $100 \mathrm{fs}$ incident light pulses with three different peak intensities. Each spectrum is normalized to the maximum value around the fundamental frequency. The corresponding temporal evolution of the graphene-induced current is shown in Fig. 2c. Remarkably, high harmonics up to 13th order are clearly discernible in the emission spectrum even at a relatively low incident peak intensity $I_{0}=10^{12} \mathrm{~W} \mathrm{~m}^{-2}$. The agreement between MDF-CEM and TB-RPA descriptions is then excellent both in the spectra (Fig. 2b, upper plots) and in the time-resolved induced current (Fig. 2c). The temporal evolution of the induced current tends to follow the profile of the incident Gaussian pulse, although a small time delay of the peak current is observed in the atomistic simulations due to the self-consistent Coulomb interaction, which persists beyond the duration of the pulse on a timescale determined by the inelastic relaxation time $\tau=13.2 \mathrm{fs}$. By raising the peak intensity, the conversion efficiency of high-order harmonics drastically increases in the MDF-CEM picture, while a more modest, yet impressive, enhancement is predicted in the atomistic TB-RPA simulations. Finite-size effects that are included in the atomistic simulations but not in the MDF-CEM description (see Methods) contribute to this discrepancy. In addition, the plasmonic localfield enhancement is self-consistently described in the TB-RPA approach, but not in the MDF-CEM method. For the high level of doping under consideration, intraband electronic transitions dominate the optical response, particularly at low intensities, while interband transitions reduce the level of anharmonicity, as observed in the temporal profiles of the induced current when comparing MDF-CEM simulations with (center plots) and without (left plots) inclusion of interband processes (Fig. 2c). The quenching of the graphene anharmonicity provided by interband
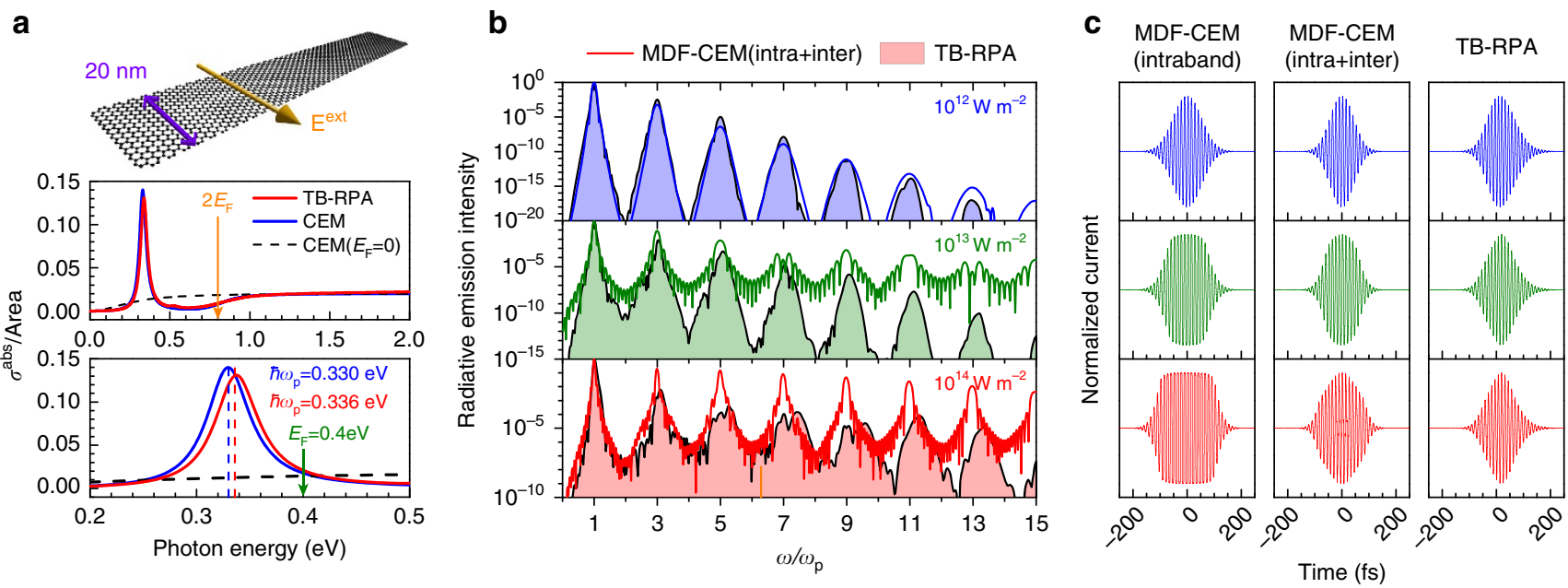

Figure 2 | HHG from a graphene nanoribbon. (a) Absorption cross-section of a 20-nm-wide armchaired graphene nanoribbon doped to a Fermi energy $E_{\mathrm{F}}=0.4 \mathrm{eV}$, as predicted by atomistic (TB-RPA, red curves) and classical electrodynamic (local-RPA conductivity at $T=300 \mathrm{~K}$, blue curves) simulations for transverse light polarization (see upper inset). The prominent resonant feature (see detail in the lower panel) corresponds to the transverse dipolar plasmon within the $2 E_{\mathrm{F}}$ optical gap. The dashed curve shows the classical simulation for the undoped ribbon. (b) Spectral decomposition of the light emission energy under illumination by a normally incident Gaussian pulse (100 fs full-width at half-maximum (FWHM) duration, centered at the frequency $\omega_{\mathrm{p}}$ of the ribbon plasmon), as calculated in the time-domain for three different pulse peak intensities (see labels) within the atomistic TB-RPA (filled curves, $\hbar \omega_{\mathrm{p}}=0.336 \mathrm{eV}$ ) and MDF-CEM (unfilled curves, $\hbar \omega_{\mathrm{p}}=0.330 \mathrm{eV}$ ) descriptions. Each curve is normalized to its own maximum value around the fundamental frequency. (c) Temporal evolution of the induced currents corresponding to the plots in $\mathbf{b}$. 

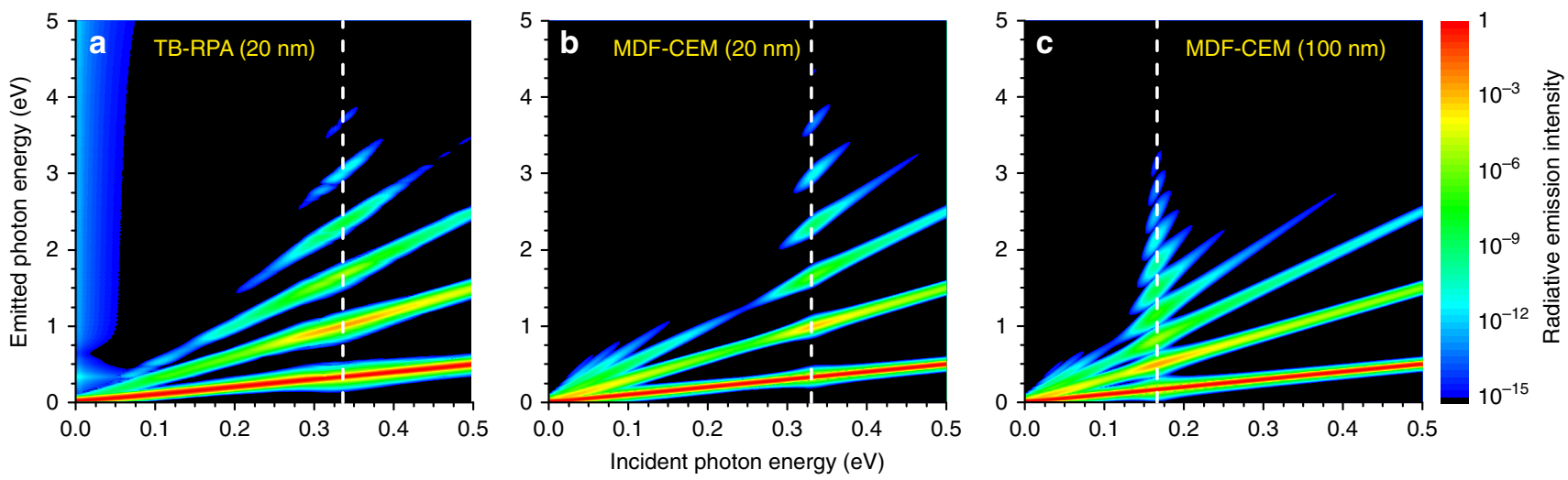

Figure 3 | Dependence of HHG on incident photon energy in graphene nanoribbons. We show the emission intensity from doped graphene nanoribbons under transverse normal illumination as a function of the incident and emitted photon energies as calculated within the (a) TB-RPA and (b,c) MDF-CEM approaches. The incident pulse has a FWHM duration of $100 \mathrm{fs}$ and a peak intensity of $10^{12} \mathrm{Wm}^{-2}$, while the Fermi energy is $0.4 \mathrm{eV}$ in all cases. The ribbon width is $20 \mathrm{~nm}$ in $\mathbf{a}$ and $\mathbf{b}$, and $100 \mathrm{~nm}$ in $\mathbf{c}$, giving rise to the plasmon energies indicated by the vertical dashed lines.

transitions results in an overall suppression of HHG, although the interband contribution is found to be marginal compared with that of the intraband response (Supplementary Fig. 1).

The marked increase in HHG from localized plasmons in graphene nanoribbons is clearly shown in Fig. 3 by mapping the emission intensity over a wide range of input pulse carrier frequencies, where at each input frequency the response is normalized to its respective maximum at the fundamental harmonic. Noticeable enhancement in harmonic generation appears when the excitation frequencies coincide with the plasmon resonance, which can be tuned actively via electrostatic gating and passively by selecting different ribbon widths. Although yet high-order harmonics appear in the spectra, we restrict our investigation to low photon energies, for which the tight-binding model for graphene remains valid (that is, below the $\pi$ plasmon near $5 \mathrm{eV}$ ). In Fig. $3 \mathrm{a}, \mathrm{b}$ we present results for the doped $20 \mathrm{~nm}$ ribbon considered previously, based on atomistic TB-RPA and MDF-CEM simulations, respectively, for $100 \mathrm{fs}$ pulses with $10^{12} \mathrm{~W} \mathrm{~m}^{-2}$ peak intensity as those considered in the upper panel of Fig. 2b. While atomistic simulations quickly become computationally unaffordable for ribbons wider than a few tens of nanometres, the MDF-CEM approach enables the exploration of HHG in much larger structures, such as the $100 \mathrm{~nm}$ wide ribbon explored in Fig. 3c, which is found to generate plasmon-enhanced high-order harmonics with superior efficiency than the $20 \mathrm{~nm}$ ribbons. The red-shifted plasmon resonances found in larger graphene nanostructures naturally lead to higher optical nonlinearities due to their increased proximity to the Dirac point ${ }^{30}$.

Even-ordered HHG from doped nanoislands. Although graphene possesses a centrosymmetric crystal lattice, the geometry of a finite nanostructure can be chosen in a manner that breaks inversion symmetry, enabling even-ordered nonlinear response in certain directions. In Fig. 4, we present atomistic TB-RPA simulations of HHG in an armchair-edged $15 \mathrm{~nm}$ equilateral graphene nanotriangle for incident light polarized normal to one of the triangle sides. When the nanotriangle is doped to a Fermi energy $E_{\mathrm{F}}=0.4 \mathrm{eV}$ and illuminated with pulses resonant with the dominant, low-energy plasmon mode (Fig. 4b), high harmonics of both even and odd orders are generated with a similar efficiency to the previously considered graphene nanoribbon (cf. Figs $3 \mathrm{a}$ and $4 \mathrm{~b}$ ). Despite the inversion symmetry of the atomic lattice, a nonzero even-order nonlinear current is produced by a combination of the strong local-field-intensity gradient and the relatively high Fermi wavelength $\lambda_{\mathrm{F}} \sim 10 \mathrm{~nm}$ (ref. 53), which is commensurate with the size of the triangle. In contrast, only oddordered harmonics appear if the nanoisland is undoped (Fig. 4a), as both of these effects (field gradient and long $\lambda_{\mathrm{F}}$ ) are then absent. In the undoped nanotriangle, a relative increase in harmonic generation appears at low energies, which we attribute to an overlap of the generated harmonic frequency with higher-energy electron-hole transitions in the discrete electronic spectrum of the nanotriangle (Supplementary Fig. 2). This is in contrast to the clear signature of plasmonic enhancement that appears when the nanotriangle is doped, which is associated with a single, dominant spectral region of increased harmonic emission.

Comparison with bulk semiconductors. Ultimately, we are interested in producing intense high harmonics using moderate incident intensities. With this goal in mind, we analyse the performance of graphene for HHG in Fig. 5 and also compare the results with available experiments in solid-state systems. As a first observation, even without the involvement of plasmons, the strong intrinsic nonlinearity of graphene is capitalized in a large relative intensity of high harmonics normalized to the response at the fundamental frequency (Fig. 5a): the relative harmonic emission reaches the values measured in GaSe samples, but using 3-4 orders of magnitude lower pulse fluence. It should be noted that a level of theory similar to the MDF model produces excellent agreement with experiment in GaSe (cf. open and solid triangles in Fig. 5a), thus supporting the predictability of our results, which is also emphasized by the agreement between MDF-CEM and atomistic simulations shown in Figs 2 and 3. By patterning the graphene into ribbons and tuning the incident light to the dominant dipole plasmon energy, HHG is boosted even more, a result that is particularly evident when analysing the absolute harmonic intensity of resonant ribbons and extended graphene (Fig. 5b). Incidentally, in contrast to the enhancement observed in doped ribbons by exciting the plasmons, doping is detrimental in extended graphene because the Fermi level is then situated in a region where the difference between parabolic and linear electronic band dispersions is reduced, and so is the nonlinear response. In Fig. 5b, we observe a saturation of harmonic generation for large peak pulse intensities, where the intensity threshold increases slightly with harmonic order. This phenomenon can be attributed to higher-order Kerr-like nonlinear processes $^{31}$, where the harmonic $s \omega$ generated by an $s$-order process is modified by the nonlinear mixing of a process at order $s^{\prime}>s$ that also generates a frequency $s \omega$. 

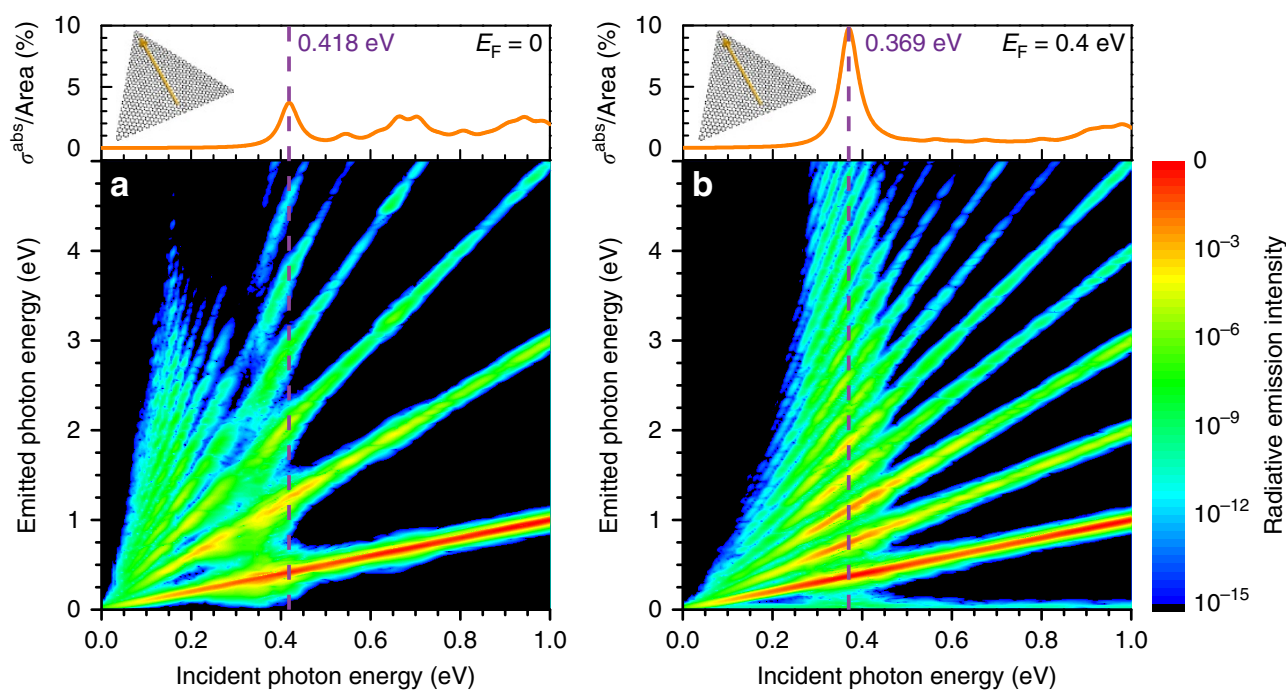

Figure 4 | HHG from triangular nanographenes. We show the emission intensity from doped graphene nanotriangles (armchair edges, equilateral $15 \mathrm{~nm}$ side length) as a function of incident and emitted photon energies, calculated within the TB-RPA approach upon pulse irradiation (100 fs FWHM duration, $10^{12} \mathrm{~W} \mathrm{~m}^{-2}$ peak intensity). We consider (a) undoped and (b) doped $\left(E_{\mathrm{F}}=0.4 \mathrm{eV}\right)$ triangles. The emission intensity in both contour plots is normalized to the overall maximum value of Fig. $4 \mathrm{~b}$ (Supplementary Fig. 2 shows data normalized to the maximum for each incident photon energy). The upper plots show the linear absorption spectrum and the orientation of the normally incident light polarization (insets).
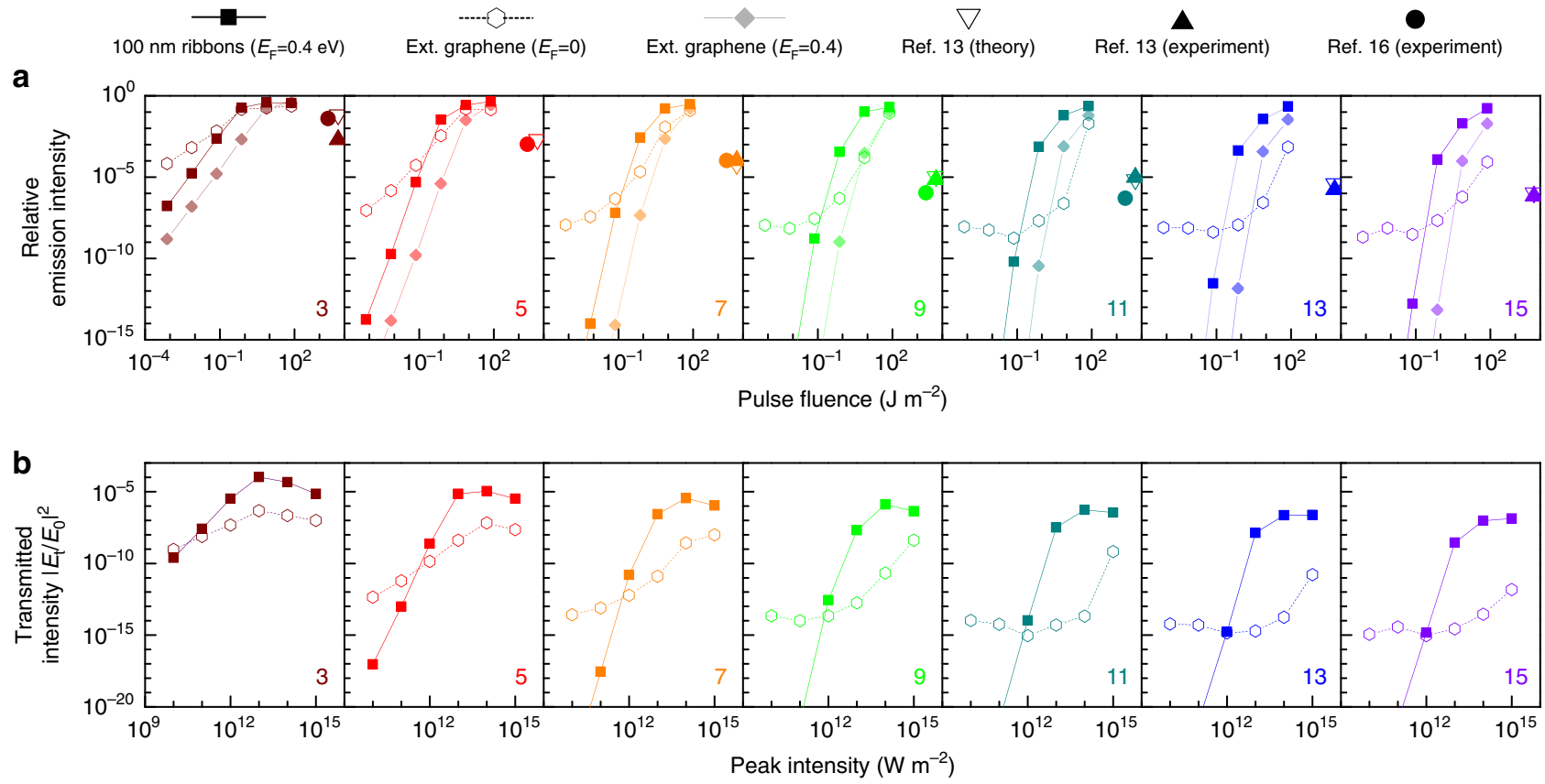

Figure 5 | Comparison of calculated graphene plasmon-assisted HHG and measured HHG from bulk semiconductors. (a) We show the emission intensities of high harmonics normalized to the intensity of the fundamental peak as a function of pulse fluence. Each panel corresponds to the emission of the harmonic indicated in its lower-right corner, calculated with the MDF-CEM method either for a $100 \mathrm{~nm}$-wide graphene nanoribbon doped to $E_{F}=0.4 \mathrm{eV}$ (solid squares) or for undoped ( $E_{F}=0$, open hexagons) and doped ( $E_{F}=0.4 \mathrm{eV}$, solid diamonds) extended graphene. For comparison, we also plot theoretical (open symbols) and experimental (solid symbols) results for bulk GaSe taken from ref. 13 (triangles) and ref. 16 (circles). (b) Comparison of HHG transmission intensity $\left(\propto\left|E_{\mathrm{t}}\right|^{2}\right)$ normalized to the incident intensity $\left(\propto\left|E_{0}\right|^{2}\right)$ for a doped-graphene ribbon array $(100 \mathrm{~nm}$ width, $200 \mathrm{~nm}$ period, $E_{\mathrm{F}}=0.4 \mathrm{eV}$, solid squares) and undoped extended graphene (open hexagons), calculated with the MDF-CEM method at the peak harmonic frequencies. We consider $100 \mathrm{fs}$ FWHM pulses centered at $0.158 \mathrm{eV}$ (that is, the $100 \mathrm{~nm}$ ribbon plasmon) in all cases.

\section{Discussion}

In summary, we predict that the combination of high intrinsic nonlinearity and strong plasmonic field confinement provided by doped graphene nanostructures under resonant illumination leads to unprecedentedly high HHG conversion efficiencies. Despite the fact that this material is only one atom thick, we show that it outperforms other solid-state systems, such as GaSe, for which HHG measurements have been reported. Among 2D materials, graphene hosts plasmons with longer lifetimes, although recent reports of large nonlinearities in transition-metal dichalcogenides ${ }^{54,55}$ warrant further investigation on the synergy between plasmon-field enhancement and intrinsic optical nonlinearities in alternative $2 \mathrm{D}$ systems. It should be noted that our results are based on a conservative value of the 
phenomenological electronic relaxation time $\tau$. The availability of high-quality graphene samples, in which $\tau$ is an order of magnitude longer, should boost HHG in this material even further (Supplementary Fig. 3). We have focused on relatively low fundamental frequencies, so that the high harmonic energies under consideration still lie within a range for which the optical response is dominated by the $\pi$ band of graphene. At low intensities, the response is well described by the low-energy, linear-dipersion region of the electronic band, which explains the agreement that we find between continuum MDF-CEM and atomistic TB-RPA descriptions. Although future work is required to extend these results to higher photon energies, which are expected to involve deeper electron bands, we conclude that the HHG conversion efficiencies associated with localized plasmons in graphene nanostructures are remarkably high for an atomic layer, indicating a strong potential for developing electrically tunable, ultra-compact nonlinear photonic devices.

\section{Methods}

TB-RPA simulations. We follow a previously reported atomistic approach ${ }^{43,47,56}$ to simulate the nonlinear optical response of graphene nanostructures via direct time-domain integration of the single-electron density matrix equation of motion,

$$
\dot{\rho}=-\frac{\mathrm{i}}{\hbar}\left[H_{\mathrm{TB}}-e \phi, \rho\right]-\frac{1}{2 \tau}\left(\rho-\rho^{0}\right),
$$

where $H_{\mathrm{TB}}$ is a tight-binding Hamiltonian describing the one-electron states of the $\pi$ band of graphene (one out-of-plane p orbital per carbon site with nearestneighbour hopping energy of $2.8 \mathrm{eV}$ ), $\phi$ is the self-consistent electric potential including both the impinging light and the Hartree interaction, and a phenomenological relaxation is assumed to bring the system to the relaxed state $\rho^{0}$ at a rate $\tau^{-1}$ with $\hbar \tau^{-1}=50 \mathrm{meV}$ (that is, the relaxation time is $\tau \approx 13.2 \mathrm{fs}$ ). The density matrix $\rho=\sum_{i j^{\prime}} \rho_{i j^{\prime}}|j\rangle\left\langle j^{\prime}\right|$ is expressed in the basis set of one-electron eigenstates of $H_{\mathrm{TB}}$, where $\rho_{j j^{\prime}}$ are the sought-after time-dependent expansion coefficients. In particular, we have $\rho_{i j^{\prime}}^{0}=\delta_{j j^{\prime}} f_{j}$ for the relaxed state, where $f_{j}$ are Fermi-Dirac occupation numbers at the initial temperature $T=300 \mathrm{~K}$. For ribbons, the states are treated as Bloch waves, arranged in bands as a function of their momentum along the direction of translational invariance, and the calculation is simplified by the orthogonality of different bands ${ }^{47}$. The induced charge density at each carbon atom position $\mathbf{R}_{l}$ is then constructed as $\rho_{l}^{\text {ind }}=-2 e \sum_{i i^{\prime}} \rho_{i j^{\prime}} a_{j l} l_{j^{\prime}}^{*}$, where the factor of 2 originates in spin degeneracy, while the coefficients $a_{j l}$ represent the change of basis set between state $j$ and site $l$ representations. Finally, the time-dependent induced dipole and surface current are given by $\mathbf{d}(t)=$ $\sum_{l} \mathbf{R}_{l} \rho_{l}^{\text {ind }}(t)$ and $\mathbf{J}(t)=\dot{\mathbf{d}}(t)$, respectively. For ribbons, we normalize these quantities per unit of ribbon length ${ }^{47}$.

MDF-CEM simulations. In a complementary approach, we model electron dynamics in graphene within the MDF picture by adopting a nonperturbative semi-analytical model ${ }^{57}$, in which light-matter interaction is introduced through the electron quasi-momentum $\boldsymbol{\pi}=\mathbf{p}+(e / c) \mathbf{A}$, where $\mathbf{p}$ is the unperturbed electron momentum, $\mathbf{A}(t)=-c \int_{-\infty}^{t} \mathbf{E}\left(t^{\prime}\right) \mathrm{d} t^{\prime}$, and $\mathbf{E}$ is the classically calculated in-plane electric field (see 'Classical electromagnetic simulations'). Electron dynamics is governed by the Dirac equation for massless fermions, which can be recast in the form of Bloch equations as ${ }^{30,31,57}$

$$
\begin{aligned}
& \dot{\Gamma}_{\mathbf{p}}=-\left(\frac{1}{\tau}+2 i \omega_{0}\right) \Gamma_{\mathbf{p}}-\frac{i e p_{y}}{p^{2}} \mathbf{E}(\mathbf{R}, t) n_{\mathbf{p}}, \\
& \dot{n}_{\mathbf{p}}=-\frac{1}{\tau}\left(n_{\mathbf{p}}+1\right)+\frac{4 e p_{y}}{p^{2}} \mathbf{E}(\mathbf{R}, t) \operatorname{Im}\left\{\Gamma_{p}\right\},
\end{aligned}
$$

where $n_{\mathbf{p}}(\mathbf{R}, t)$ and $\Gamma_{\mathbf{p}}(\mathbf{R}, t)$ represent the population inversion and the interband coherence, respectively ${ }^{57}$. Here, the damping energy $\hbar \tau^{-1}=50 \mathrm{meV}$ is the same as in the TB-RPA approach. These equations describe both inter- and intraband transitions. We solve equations ( $2 \mathrm{a})$ and $(2 \mathrm{~b})$ nonperturbatively under the slowly varying envelope approximation ${ }^{57}$ by expanding $\Gamma_{\mathbf{p}}(\mathbf{R}, t)=\sum_{j=0}^{N}\left[\Gamma_{\mathbf{p}, j}^{+}\right.$ $\left.(\mathbf{R}, t) e^{i(2 j+1) \omega t}+\Gamma_{\mathbf{p}, j}^{-}(\mathbf{R}, t) e^{-i(2 j+1) \omega t}\right]$ and $n_{\mathbf{p}}(\mathbf{R}, t)=n_{0}(\mathbf{R})+\sum_{j=1}^{N} \operatorname{Re}\left[n_{\mathbf{p}, j}^{+}\right.$, $\left.(\mathbf{R}, t) e^{2 i j \omega t}+n_{\mathbf{p}, j}^{-}(\mathbf{R}, t) e^{-2 i j \omega t}\right]$ in harmonic series up to $N=15$. The current is then parallel to the local electric field $\mathbf{E}(\mathbf{R}, t) \| \hat{\mathbf{x}}$, while its amplitude is calculated as an integral over momentum-resolved contributions,

$$
J(\mathbf{R}, t)=-\frac{e v_{\mathrm{F}}}{\pi^{2} \hbar^{2}} \int \mathrm{d}^{2} \mathbf{p}\left[\frac{p_{x}+e A}{\sqrt{\left(p_{x}+e A\right)^{2}+p_{y}^{2}}}\left(n_{\mathbf{p}}+1\right)-\frac{2 p_{y}}{p} \operatorname{Im}\left\{\Gamma_{\mathbf{p}}\right\}\right] .
$$

Finally, the far-field power spectrum of the emitted light is proportional to $|\omega\langle\mathbf{J}(\mathbf{R}, \omega)\rangle|^{2}$, where $J(\mathbf{R}, \omega)$ is the time-Fourier transform of $\mathbf{J}(\mathbf{R}, t)$, and $\langle\ldots\rangle$ denotes the space average over the graphene structure under examination.
Classical electromagnetic simulations. The classical response of graphene nanostructures is simulated by numerically solving Maxwell's equations using the boundary-element method ${ }^{58}$ for ribbons and a finite-element method (COMSOL) for triangles. We describe graphene as a thin film (thickness $s=0.5 \mathrm{~nm})$ of permittivity $\epsilon(\omega)=1+4 \pi i \sigma(\omega) / \omega s$, where $\sigma(\omega)$ is the local-RPA conductivity $40,59,60$. We thus obtain the linear optical extinction and the near-field distribution. Given the small lateral size of the ribbons and triangles compared with the light wavelength, we adopt a quasistatic eigenmode expansion ${ }^{61}$ and only retain one term corresponding to the dominant plasmon in each case. The incident light pulses are taken to have a large duration compared with the optical cycle, so we approximate them by a single carrier frequency (the pulse peak frequency) times a Gaussian envelope. We also use this approximation for the input near-field $\mathbf{E}$ of the MDF-CEM approach, with the carrier component classically calculated as explained above.

Data availability. The data that support the findings of this study are available from the corresponding authors upon request.

\section{References}

1. Ferray, M. et al. Multiple-harmonic conversion of $1,064 \mathrm{~nm}$ radiation in rare gases. J. Phys. B 419, 803-807 (1988).

2. L'Huillier, A. \& Balcou, P. High-order harmonic generation in rare gases with a 1-ps 1053-nm laser. Phys. Rev. Lett. 70, 774-777 (1993).

3. Priori, E. et al. Nonadiabatic three-dimensional model of high-order harmonic generation in the few-optical-cycle regime. Phys. Rev. A 61, 063801 (2000).

4. Spielmann, C. et al. Generation of coherent X-rays in the water window using 5-femtosecond laser pulses. Science 278, 661-664 (1997).

5. Brabec, T. \& Krausz, F. Intense few-cycle laser fields: Frontiers of nonlinear optics. Rev. Mod. Phys. 72, 545-591 (2000).

6. Goulielmakis, E. et al. Single-cycle nonlinear optics. Science 320, 1614-1617 (2008).

7. Paul, P. M. et al. Observation of a train of attosecond pulses from high harmonic generation. Science 292, 1689-1692 (2001).

8. Corkum, P. B. \& Krausz, F. Attosecond science. Nat. Phys. 3, 381-387 (2007).

9. Krausz, F. \& Ivanov, M. Attosecond physics. Rev. Mod. Phys. 81, 163-234 (2009).

10. Itatani, J. et al. Tomo-graphic imaging of molecular orbitals. Nature 432, 867-871 (2004).

11. Wörner, H. J., Bertrand, J. B., Kartashov, D. V., Corkum, P. B. \& Villeneuve, D. M. Following a chemical reaction using high-harmonic interferometry. Nature 466, 604-607 (2010).

12. Ghimire, S. et al. Observation of high-order harmonic generation in a bulk crystal. Nat. Phys. 7, 138-141 (2011).

13. Schubert, O. et al. Sub-cycle control of terahertz high-harmonic generation by dynamical Bloch oscillations. Nat. Photon. 8, 119-123 (2014).

14. Luu, T. T. et al. Extreme ultraviolet high-harmonic spectroscopy of solids. Nature 521, 498-502 (2015).

15. Vampa, G. et al. Linking high harmonics from gases and solids. Nature 522, 462-464 (2015).

16. Hohenleutner, M. et al. Realtime observation of interfering crystal electrons in high-harmonic generation. Nature 523, 572-575 (2015).

17. Corkum, P. B. Plasma perspective on strong-field multiphoton ionization. Phys Rev. Lett. 71, 1994-1997 (1993).

18. Lewenstein, M., Balcou, P., Ivanov, M. Y., L’Huillier, A. \& Corkum, P. B. Theory of high-harmonic generation by low-frequency laser fields. Phys. Rev. A 49, 2117-2132 (1994).

19. Tamaya, T., Ishikawa, A., Ogawa, T. \& Tanaka, K. Diabatic mechanisms of higher-order harmonic generation in solid-state materials under high-intensity electric fields. Phys. Rev. Lett. 116, 016601 (2016).

20. Osika, E. N. et al.Wannier-Bloch approach to localization in high harmonics generation in solids. Preprint at arXiv:1607.07622 (2016).

21. Tancogne-Dejean, N., Mücke, O. D., Kärtner, F. X. \& Rubio, A. Impact of the electronic band structure in high-harmonic generation spectra of solids. Preprint at arXiv:1609.09298 (2016).

22. Wallace, P. R. The band theory of graphite. Phys. Rev. 71, 622-634 (1947).

23. Geim, A. K. \& Novoselov, K. S. The rise of graphene. Nat. Mater. 6, 183-191 (2007).

24. Hendry, E., Hale, P. J., Moger, J., Savchenko, A. K. \& Mikhailov, S. A. Coherent nonlinear response of graphene. Phys. Rev. Lett. 105, 097401 (2010).

25. Wu, R. et al. Purely coherent nonlinear optical response in solution dispersion of graphene sheets. Nano Lett. 11, 5159-5164 (2011).

26. Gu, T. et al. Regenerative oscillation and four-wave mixing in grapheme optoelectronics. Nat. Photon. 6, 554-559 (2012).

27. Kumar, N. et al. Third harmonic generation in graphene and few-layer graphite films. Phys. Rev. B 87, 121406(R) (2013).

28. Hong, S.-Y. et al. Optical third-harmonic generation in graphene. Phys. Rev. X 3, 021014 (2013) 
29. An, Y. Q., Rowe, J. E., Dougherty, D. B., Lee, J. U. \& Diebold, A. C. Optical second-harmonic generation induced by electric current in graphene on $\mathrm{Si}$ and SiC substrates. Phys. Rev. B 89, 115310 (2014).

30. Ishikawa, K. L. Nonlinear optical response of graphene in time domain. Phys. Rev. B 82, 201402(R) (2010).

31. Al-Naib, I., Sipe, J. E. \& Dignam, M. M. High harmonic generation in undoped graphene: Interplay of inter- and intraband dynamics. Phys. Rev. B 90, 245423 (2014).

32. Paul, M. J. et al. High-field terahertz response of graphene. New J. Phys. 15, 085019 (2013).

33. Bowlan, P., Martinez-Moreno, E., Reimann, K., Elsaesser, T. \& Woerner, M. Ultrafast terahertz response of multilayer graphene in the nonperturbative regime. Phys. Rev. B 89, 041408(R) (2014).

34. Jablan, M., Buljan, H. \& Soljačić, M. Plasmonics in graphene at infrared frequencies. Phys. Rev. B 80, 245435 (2009).

35. Fei, Z. et al. Infrared nanoscopy of Dirac plasmons at the graphene-SiO interface. Nano Lett. 11, 4701-4705 (2011).

36. Koppens, F. H. L., Chang, D. E. \& García de Abajo, F. J. Graphene plasmonics: A platform for strong light-matter interactions. Nano Lett. 11, 3370-3377 (2011).

37. Chen, J. et al. Optical nano-imaging of gate-tunable graphene plasmons. Nature 487, 77-81 (2012).

38. Fei, Z. et al. Gate-tuning of graphene plasmons revealed by infrared nanoimaging. Nature 487, 82-85 (2012).

39. Grigorenko, A. N., Polini, M. \& Novoselov, K. S. Graphene plasmonics. Nat Photon. 6, 749-758 (2012)

40. García de Abajo, F. J. Graphene plasmonics: Challenges and opportunities. ACS Photon 1, 135-152 (2014).

41. Mikhailov, S. A. Non-linear electromagnetic response of graphene. Europhys. Lett. 79, 27002 (2007)

42. Mikhailov, S. A. Quantum theory of the third-order nonlinear electrodynamic effects of graphene. Phys. Rev. B 93, 085403 (2016).

43. Cox, J. D. \& García de Abajo, F. J. Electrically tunable nonlinear plasmonics in graphene nanoislands. Nat. Commun. 5, 5725 (2014).

44. Jablan, M. \& Chang, D. E. Multiplasmon absorption in graphene. Phys. Rev. Lett. 114, 236801 (2015).

45. Cheng, J. L., Vermeulen, N. \& Sipe, J. E. Third-order nonlinearity of graphene: Effects of phenomenological relaxation and finite temperature. Phys. Rev. B 91, 235320 (2015).

46. Christensen, T., Yan, W., Jauho, A.-P., Wubs, M. \& Mortensen, N. A. Kerr nonlinearity and plasmonic bistabil-ity in graphene nanoribbons. Phys. Rev. B 92, 121407(R) (2015).

47. Cox, J. D., Silveiro, I. \& García de Abajo, F. J. Quantum effects in the nonlinear response of graphene plasmons. ACS Nano 10, 1995-2003 (2016).

48. Kim, S. et al. High-harmonic generation by resonant plasmon field enhancement. Nature 453, 757-760 (2008).

49. Park, I.-Y. et al. Plasmonic generation of ultrashort extreme-ultraviolet light pulses. Nat. Photon 5, 677-681 (2011).

50. Husakou, A., Im, S.-J. \& Herrmann, J. Theory of plasmonenhanced high-order harmonic generation in the vicinity of metal nanostructures in noble gases. Phys. Rev. A 83, 043839 (2011).

51. Tikman, Y. et al. High-order-harmonic generation from Rydberg atoms driven by plasmon-enhanced laser fields. Phys. Rev. A 93, 023410 (2016).

52. Brey, L., Johnson, N. F. \& Halperin, B. I. Optical and magneto-optical absorption in parabolic quantum wells. Phys. Rev. B 40, 10647-10649 (1989).

53. Manzoni, M. T., Silveiro, I., García de Abajo, F. J. \& Chang, D. E. Second-order quantum nonlinear optical processes in single graphene nanostructures and arrays. New J. Phys. 17, 083031 (2015).
54. Yin, X. et al. Edge nonlinear optics on a $\mathrm{MoS}_{2}$ atomic monolayer. Science 344, 448-490 (2014).

55. Wang, C.-Y. \& Guo, G.-Y. Nonlinear optical properties of transition-metal dichalcogenide MX2 $(\mathrm{M}=\mathrm{Mo}, \mathrm{W} ; \mathrm{X}=\mathrm{S}, \mathrm{Se})$ monolayers and trilayers from first-principles calculations. J. Phys. Chem. C 119, 13268-13276 (2015).

56. Thongrattanasiri, S., Manjavacas, A. \& García de Abajo, F. J. Quantum finite-size effects in graphene plasmons. ACS Nano 6, 1766-1775 (2012).

57. Marini, A., Cox, J. D. \& García de Abajo, F. J. Theory of graphene saturable absorption. Preprint at arXiv:1605.06499 (2016).

58. García de Abajo, F. J. \& Howie, A. Retarded field calculation of electron energy loss in inhomogeneous dielectrics. Phys. Rev. B 65, 115418 (2002).

59. Gusynin, V. P., Sharapov, S. G. \& Carbotte, J. P. Unusual microwave response of dirac quasiparticles in graphene. Phys. Rev. Lett. 96, 256802 (2006).

60. Gusynin, V. P., Sharapov, S. G. \& Carbotte, J. P. On the universal ac optical background in graphene. New J. Phys. 11, 095013 (2009).

61. García de Abajo, F. J. Multiple excitation of confined graphene plasmons by single free electrons. ACS Nano 7, 11409-11419 (2013)

\section{Acknowledgements}

We thank Jens Biegert and Fernando Sols for stimulating and enjoyable discussions and Renwen $\mathrm{Yu}$ for providing the plasmon wave function for triangles and the resonant near-field for ribbons. This work has been supported in part by the Spanish MINECO (MAT2014-59096-P and SEV2015-0522), AGAUR (2014 SGR 1400), Fundació Privada Cellex, and the European Commission (Graphene Flagship CNECT-ICT-604391 and FP7-ICT-2013-613024-GRASP).

\section{Author contributions}

F.J.G.d.A. proposed the study. J.D.C., A.M., and F.J.G.d.A. worked out the theory, discussed the results, and wrote the paper. J.D.C. and A.M. performed the numerical calculations.

\section{Additional information}

Supplementary Information accompanies this paper at http://www.nature.com/ naturecommunications

Competing financial interests: The authors declare no competing financial interests

Reprints and permission information is available online at http://npg.nature.com/ reprintsandpermissions/

How to cite this article: Cox, J. D. et al. Plasmon-assisted high-harmonic generation in graphene. Nat. Commun. 8, 14380 doi: 10.1038/ncomms14380 (2017).

Publisher's note: Springer Nature remains neutral with regard to jurisdictional claims in published maps and institutional affiliations.

This work is licensed under a Creative Commons Attribution 4.0 International License. The images or other third party material in this article are included in the article's Creative Commons license, unless indicated otherwise in the credit line; if the material is not included under the Creative Commons license, users will need to obtain permission from the license holder to reproduce the material. To view a copy of this license, visit http://creativecommons.org/licenses/by/4.0/

(C) The Author(s) 2017 